\title{
A System of Two Diophantine Inequalities with Primes
}

\author{
Xue Han, Huafeng Liu $(\mathbb{D}$, and Deyu Zhang \\ School of Mathematics and Statistics, Shandong Normal University, Jinan, Shandong 250358, China \\ Correspondence should be addressed to Huafeng Liu; hfliu_sdu@hotmail.com
}

Received 2 January 2021; Revised 26 January 2021; Accepted 29 January 2021; Published 15 February 2021

Academic Editor: Jie Wu

Copyright ( $\odot 2021$ Xue Han et al. This is an open access article distributed under the Creative Commons Attribution License, which permits unrestricted use, distribution, and reproduction in any medium, provided the original work is properly cited.

Let $1<d<c<128 / 119,1<\alpha<\beta<6^{1-d / c}$. In this paper, we prove that there exist positive real numbers $N_{1}^{(0)}$ and $N_{2}^{(0)}$ depending on $c, d, \alpha, \beta$ such that for all real numbers $N_{1}>N_{1}^{(0)}, N_{2}>N_{2}^{(0)}$ and $\alpha \leq N_{2} / N_{1}^{d / c} \leq \beta$, the system of two Diophantine inequalities $\mid p_{1}^{c}+$ $\cdots+p_{6}^{c}-N_{1}\left|<N_{1}^{-(1 / c)(128 / 119-c)} \log ^{109} N_{1},\right| p_{1}^{d}+\cdots+p_{6}^{d}-N_{2} \mid<N_{2}^{-(1 / d)(128 / 119-d)} \log ^{109} N_{2}$ is solvable in prime variables $p_{1}, \ldots, p_{6}$.

\section{Introduction}

Assume that $c>1$ is not an integer and $\varepsilon$ is a sufficiently small positive number. Let $H(c)$ denote the least integer $s$ such that the Diophantine inequality

$$
\left|p_{1}^{c}+p_{2}^{c}+\cdots+p_{s}^{c}-N\right|<\varepsilon
$$

is solvable in primes $p_{1}, p_{2}, \ldots, p_{s}$ for sufficiently large $N$. In 1952, Piatetski-Shapiro [1] proved that

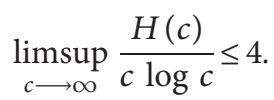

Piatetski-Shapiro also proved $H(c) \leq 5$ for $1<c<3 / 2$. Tolev [2] first improved this result for $c$ close to one. More precisely, Tolev proved that if $1<c<15 / 14^{6}$, the Diophantine inequality

$$
\left|p_{1}^{c}+p_{2}^{c}+p_{3}^{c}-N\right|<\varepsilon(N)
$$

has prime solutions $p_{1}, p_{2}, p_{3}$ for large $N$, where $\varepsilon(N)=N^{-(1 / c)(15 / 14-c)} \log ^{9} N$. Later, this result was improved by many authors (see [3-9]), and many analogous problems of this type were studied (for example, see [10-17]).

In 1995, Tolev [18] first considered the system of two Diophantine inequalities with primes

$$
\begin{aligned}
& \left|p_{1}^{c}+\cdots+p_{5}^{c}-N_{1}\right|<\varepsilon_{1}^{*}\left(N_{1}\right), \\
& \left|p_{1}^{d}+\cdots+p_{5}^{d}-N_{2}\right|<\varepsilon_{2}^{*}\left(N_{2}\right) .
\end{aligned}
$$

He established that for all real numbers $N_{1}, N_{2}$ satisfying $N_{1}>N_{1}^{(0)}, N_{2}>N_{2}^{(0)}$ and $\alpha \leq N_{2} / N_{1}^{d / c} \leq \beta$, system (4) with

$$
\begin{aligned}
\varepsilon_{1}^{*}\left(N_{1}\right) & =N_{1}^{-(1 / c)(35 / 34-c)} \log ^{12} N_{1}, \varepsilon_{2}^{*}\left(N_{2}\right) \\
& =N_{2}^{-(1 / d)(35 / 34-d)} \log ^{12} N_{2}
\end{aligned}
$$

has solutions in primes $p_{1}, \ldots, p_{5}$, where $c, d, \alpha, \beta$ are real numbers satisfying the conditions

$$
1<d<c<35 / 34,1<\alpha<\beta<5^{1-d / c},
$$

and $N_{1}^{(0)}, N_{2}^{(0)}$ depending on $c, d, \alpha, \beta$ are sufficiently large numbers. Subsequently, Tolev's result was improved by Zhai [19] and Zhai and Cao [20]. Now the best result is due to Zhai and Cao [20] who proved that system (4) with

$$
\begin{aligned}
\varepsilon_{1}^{*}\left(N_{1}\right) & =N_{1}^{-(1 / c)(27 / 26-c)} \log ^{100} N_{1}, \varepsilon_{2}^{*}\left(N_{2}\right) \\
& =N_{2}^{-(1 / d)(27 / 26-d)} \log ^{100} N_{2}
\end{aligned}
$$

is solvable for $1<d<c<27 / 26$.

In this paper, we consider the following system of two Diophantine inequalities over primes $p_{1}, \ldots, p_{6}$ : 


$$
\begin{aligned}
& \left|p_{1}^{c}+\cdots+p_{6}^{c}-N_{1}\right|<\varepsilon_{1}\left(N_{1}\right) \\
& \left|p_{1}^{d}+\cdots+p_{6}^{d}-N_{2}\right|<\varepsilon_{2}\left(N_{2}\right)
\end{aligned}
$$

where $c>1, d>1$ are different numbers but close to 1 and $\varepsilon_{1}\left(N_{1}\right), \varepsilon_{2}\left(N_{2}\right)$ satisfy

$$
\begin{aligned}
& \varepsilon_{1}\left(N_{1}\right) \longrightarrow 0, \text { as } N_{1} \longrightarrow \infty, \\
& \varepsilon_{2}\left(N_{2}\right) \longrightarrow 0, \text { as } N_{2} \longrightarrow \infty
\end{aligned}
$$

We have to impose a condition on the orders of $N_{1}$ and $\mathrm{N}_{2}$ due to the inequality

$$
\left(x_{1}^{c}+\cdots+x_{6}^{c}\right)^{d / c} \leq x_{1}^{d}+\cdots+x_{6}^{d} \leq 6^{1-d / c}\left(x_{1}^{c}+\cdots+x_{6}^{c}\right)^{d / c},
$$

which holds for every positive $x_{1}, \ldots, x_{6}$ provided $1<d<c$. Our result is as follows.

Theorem 1. Suppose that $c, d, \alpha, \beta$ are real numbers and satisfy conditions

$$
\begin{aligned}
& 1<d<c<128 / 119, \\
& 1<\alpha<\beta<6^{1-d / c} .
\end{aligned}
$$

Then, there exist positive real numbers $N_{1}^{(0)}, N_{2}^{(0)}$ depending on $c, d, \alpha, \beta$ such that for all real numbers $N_{1}>N_{1}^{(0)}, N_{2}>N_{2}^{(0)}$ and

$$
\alpha \leq N_{2} / N_{1}^{d / c} \leq \beta,
$$

system (8) with

$$
\begin{aligned}
& \varepsilon_{1}\left(N_{1}\right)=N_{1}^{-(1 / c)(128 / 119-c)} \log ^{109} N_{1}, \\
& \varepsilon_{2}\left(N_{2}\right)=N_{2}^{-(1 / d)(128 / 119-d)} \log ^{109} N_{2}
\end{aligned}
$$

has solutions in primes $p_{1}, \ldots, p_{6}$.

Notation. Throughout this paper, the letter $p$, with or without a subscript, always represents a prime, $c$ and $d$ are real numbers satisfying (11), and $\alpha$ and $\beta$ are real numbers satisfying (12). $\eta$ denotes a sufficiently small positive number depending on $c$ and $d$. $\chi(t)$ denotes the characteristic function over the interval $[-1,1] . \rho=\beta^{\prime}+i \gamma^{\prime}$ is the nontrivial zero of the Riemann zeta function $\zeta(s)$. As usual, $\Lambda(n)$ and $\tau(n)$ are the von Mangoldt function and the divisor function, respectively. $N_{1}$ and $N_{2}$ are sufficiently large numbers. We set

$$
\begin{aligned}
X & =N_{1}^{1 / c}, \tau_{1}=X^{3 / 4-c-\eta}, \tau_{2}=X^{3 / 4-d-\eta}, \\
\varepsilon_{1} & =X^{-(128 / 119-c)} \log ^{107} X, \varepsilon_{2}=X^{-(128 / 119-d)} \log ^{107} X, \\
K_{1} & =X^{(128 / 119-c)} \log ^{-106} X, K_{2}=X^{(128 / 119-d)} \log ^{-106} X, \\
e(t) & =e^{2 \pi i t}, \varphi(t)=e^{-\pi t^{2}}, \varphi_{\delta}(t)=\delta \varphi(\delta t) .
\end{aligned}
$$

\section{Outline of the Proof}

Let $\lambda$ denote a sufficiently small positive number, whose value depends on $c, d, \alpha, \beta$ and will be determined more precisely in Lemma 1 . Let

$$
\begin{aligned}
B & =\sum_{\lambda X<p_{1}, \cdots, p_{6} \leq X}\left(\log p_{1}\right) \cdots\left(\log p_{6}\right) \chi\left(\frac{p_{1}^{c}+\cdots+p_{6}^{c}-N_{1}}{\varepsilon_{1} \log X}\right) \chi\left(\frac{p_{1}^{d}+\cdots+p_{6}^{d}-N_{2}}{\varepsilon_{2} \log X}\right), \\
S(x, y) & =\sum_{\lambda X<p \leq X}(\log p) e\left(x p^{c}+y p^{d}\right), \\
D & =\int_{-\infty}^{\infty} \int_{-\infty}^{\infty} S^{6}(x, y) e\left(-N_{1} x-N_{2} y\right) \varphi_{\varepsilon_{1}}(x) \varphi_{\varepsilon_{2}}(y) \mathrm{d} x \mathrm{~d} y .
\end{aligned}
$$

We divide the plane into three regions: the neighbourhood of origin $\Omega_{1}$, the intermediate region $\Omega_{2}$, and the trivial region $\Omega_{3}$, which are defined as

$$
\begin{aligned}
\Omega_{1}= & \left\{(x, y): \max \left(|x| / \tau_{1},|y| / \tau_{2}\right)<1\right\}, \\
\Omega_{2}= & \left\{(x, y): \max \left(|x| / \tau_{1},|y| / \tau_{2}\right) \geq 1,\right. \\
& \left.\max \left(|x| / K_{1}|y| / K_{2}\right) \leq 1\right\}, \\
\Omega_{3}= & \left\{(x, y): \max \left(|x| / K_{1}|y| / K_{2}\right)>1\right\} .
\end{aligned}
$$

Thus, the integral $D$ can be represented as

$$
D=D_{1}+D_{2}+D_{3}
$$

where

$$
\begin{gathered}
D_{i}=\iint_{\Omega_{i}} S^{6}(x, y) e\left(-N_{1} x-N_{2} y\right) \varphi_{\varepsilon_{1}}(x) \varphi_{\varepsilon_{2}}(y) \mathrm{d} x \mathrm{~d} y \\
\quad(i=1,2,3) .
\end{gathered}
$$

If we show that $B$ tends to infinity as $X$ tends to infinity, then Theorem 1 holds. From Lemma 4, it is sufficient to prove that $D$ tends to infinity as $X$ tends to infinity. To do this, noting (20), we shall prove the following:

$$
\left|D_{1}\right| \gg \varepsilon_{1} \varepsilon_{2} X^{6-c-d}
$$

$$
\left|D_{2}\right| \ll \frac{\varepsilon_{1} \varepsilon_{2} X^{6-c-d}}{\log X},
$$




$$
\left|D_{3}\right| \ll 1 .
$$

In Section 3, we first give some auxiliary lemmas. Inequality (22) is proved in Section 4. Inequality (23), from which we can get the range of $c$ and $d$, is proved in Section 5 . In Section 6, we complete the proof of Theorem 1.

\section{Auxiliary Lemmas}

Lemma 1. Let $\delta \in[\alpha, \beta]$. There exists $\lambda>0$ depending on $c, d, \alpha, \beta$ such that for the volume $V$ of the domain in sixdimensional space defined by

$$
t_{1}, \ldots, t_{6}>\lambda,\left|t_{1}^{c}+\cdots+t_{6}^{c}-1\right|<\mu_{1},\left|t_{1}^{d}+\cdots+t_{6}^{d}-\delta\right|<\mu_{2},
$$

we have

$$
V \gg \mu_{1} \mu_{2},
$$

provided $\mu_{1}, \mu_{2}$ are sufficiently small.

Proof. This lemma is similar to Lemma 1 in Tolev [18]. We can write the volume of $V$ as

$$
V=\int_{\substack{t_{1}, \ldots, t_{6}>\lambda \\\left|t_{1}^{c}+\cdots+t_{6}^{c}-1\right|<\mu_{1} \\\left|t_{1}^{d}+\cdots+t_{6}^{d}-\delta\right|<\mu_{2}}} \cdots \int t_{1} \cdots \mathrm{d} t_{6} .
$$

Then, we can fix $t_{1}, \ldots, t_{5}$ to get the range of $t_{6}$ from last two inequalities and get

$$
\begin{aligned}
& \left(1-t_{1}^{c}+\cdots+t_{5}^{c}-\mu_{1}\right)^{1 / c}<t_{6}<\left(1-t_{1}^{c}+\cdots+t_{5}^{c}+\mu_{1}\right)^{1 / c}, \\
& \left(\delta-t_{1}^{d}+\cdots+t_{5}^{d}-\mu_{2}\right)^{1 / d}<t_{6}<\left(\delta-t_{1}^{d}+\cdots+t_{5}^{d}+\mu_{2}\right)^{1 / d} .
\end{aligned}
$$

Since $\mu_{1}$ and $\mu_{2}$ are sufficiently small, we can adjust the value of $\lambda$ to ensure that there are intersections between (28) and (29). We may also get this lemma by adjusting the value of $\lambda$ and using circle method to estimate

$$
\left|t_{1}^{c}+\cdots+t_{6}^{c}-1\right|<\mu_{1},\left|t_{1}^{d}+\cdots+t_{6}^{d}-\delta\right|<\mu_{2} .
$$

Lemma 2 (see [18], Lemma 2). The function $\varphi(t)=e^{-\pi t^{2}}$ has the following properties:

$$
\begin{gathered}
\varphi(x)=\int_{-\infty}^{\infty} \varphi(t) e(-x t) \mathrm{d} t, \\
\chi(t / \varrho) \geq \varphi(t)-e^{-\pi \rho^{2}} \quad \text { for } \varrho>0, \\
\varphi(t) \geq e^{-\pi} \quad \text { for }|t| \leq 1 .
\end{gathered}
$$

Lemma 3. Let $B>1$ denote a real number and $f$ be a smooth real function on $[B, 2 B]$. Suppose that there exists a positive constant $A=A(f)$ such that

$$
\mathrm{AB}^{1-j} \ll_{j}\left|f^{j}(x)\right| \ll_{j} \mathrm{AB}^{1-j}, \quad \text { for } x \sim B \text { and } j \in \mathbb{N},
$$

where the implied absolute constant depends only on $j$. Then, there exists an exponent pair $(\kappa, l)$ with

$$
0 \leq \kappa \leq 1 / 2 \leq \iota<1 \text {, }
$$

such that

$$
\sum_{B<n \leq B+h} e(f(n)) \ll A^{\kappa} B^{l}, \quad \text { for } 1<h \leq B .
$$

Proof. We can find this lemma in Ivić ([21], pp. 72-79).

Lemma 4. The quantities $B$ and D satisfy

$$
B \geq D+O(1) .
$$

Proof. From (31) and (32) in Lemma 2, we have

$$
\begin{aligned}
& \chi\left(\frac{p_{1}^{c}+\cdots+p_{6}^{c}-N_{1}}{\varepsilon_{1} \log X}\right) \geq \varphi\left(\frac{p_{1}^{c}+\cdots+p_{6}^{c}-N_{1}}{\varepsilon_{1}}\right)-e^{-\pi(\log X)^{2}} \\
& \varphi\left(\frac{p_{1}^{c}+\cdots+p_{6}^{c}-N_{1}}{\varepsilon_{1}}\right)=\varphi\left(\frac{N_{1}-\left(p_{1}^{c}+\cdots+p_{6}^{c}\right)}{\varepsilon_{1}}\right) \\
& =\int_{-\infty}^{\infty} \varphi(x) e\left(-x \frac{N_{1}-\left(p_{1}^{c}+\cdots+p_{6}^{c}\right)}{\varepsilon_{1}}\right) \mathrm{d} x \\
& =\int_{-\infty}^{\infty} e\left(x\left(p_{1}^{c}+\cdots+p_{6}^{c}\right)\right) e\left(-x N_{1}\right) \varphi_{\varepsilon_{1}}(x) \mathrm{d} x,
\end{aligned}
$$

where we substitute $\varepsilon_{1} x$ for $x$. Similarly, we have

$$
\begin{aligned}
& \chi\left(\frac{p_{1}^{d}+\cdots+p_{6}^{d}-N_{2}}{\varepsilon_{2} \log X}\right) \geq \varphi\left(\frac{p_{1}^{d}+\cdots+p_{6}^{d}-N_{2}}{\varepsilon_{2}}\right)-e^{-\pi(\log X)^{2}} \\
& \varphi\left(\frac{p_{1}^{d}+\cdots+p_{6}^{d}-N_{2}}{\varepsilon_{2}}\right)=\int_{-\infty}^{\infty} e\left(y\left(p_{1}^{d}+\cdots+p_{6}^{d}\right)\right) e\left(-y N_{2}\right) \varphi_{\varepsilon_{2}}(y) \mathrm{d} y .
\end{aligned}
$$

Then, 


$$
\begin{aligned}
B \geq & \sum_{\substack{\lambda<p_{1}, \ldots, p_{6} \leq X\\
}}\left(\log p_{1}\right) \cdots\left(\log p_{6}\right) \int_{-\infty}^{\infty} \int_{-\infty}^{\infty} e\left(x\left(p_{1}^{c}+\cdots+p_{6}^{c}\right)+y\left(p_{1}^{d}+\cdots+p_{6}^{d}\right)\right) e\left(-x N_{1}-y N_{2}\right) \\
& \varphi_{\varepsilon_{1}}(x) \varphi_{\varepsilon_{2}}(y) \mathrm{d} x \mathrm{~d} y+O(1) \\
\geq & D+O(1) .
\end{aligned}
$$

Lemma 5. There are two real functions $G(x), F(x)$ defined in $[a, b],|G(x)| \leq H$ for $a \leq x \leq b$ and $G(x) / F(x)$ is a monotonous function. Write

$$
I=\int_{a}^{b} G(x) e(F(x)) \mathrm{d} x .
$$

If $F^{\prime}(x) \geq h>0$ or $F^{\prime}(x) \leq-h<0$ for all $x \in[a, b]$, then

$$
|I| \ll H / h \text {. }
$$

If $F^{\prime \prime}(x) \geq h>0$ for all $x \in[a, b]$, then

$$
|I| \ll H / \sqrt{h} \text {. }
$$

Proof. This lemma can be found in Ivić ([21], pp. 56-57).

Lemma 6. Let $\Psi(u)=\sum_{n \leq u} \Lambda(n)$ and $2 \leq t \leq u$; then,

$$
\Psi(u)=u-\sum_{\left|\gamma^{\prime}\right| \leq t} \frac{u^{\rho}}{\rho}+O\left(\frac{u \log ^{2} u}{t}\right),
$$

where $\rho=\beta^{\prime}+i \gamma^{\prime}$ is the nontrivial zero of $\zeta(s)$.

Proof. This is a well-known explicit formula, which can be found in Karatsuba ([22], p. 80).

\section{The Estimate of the Integral $D_{1}$}

In this section, we give the estimate of the integral $D_{1}$ and set $T=X^{3 / 4+119 c / 512-\eta}$.

Lemma 7. If $1 \leq T_{1} \leq T$, then

$$
\frac{1}{\sqrt{T_{1}}} \sum_{0<\gamma^{\prime} \leq T_{1}} X^{\beta^{\prime}} \ll X e^{-(\log X)^{1 / 4}},
$$

and

$$
\frac{1}{T_{1}} \sum_{0<\gamma^{\prime} \leq T_{1}} X^{\beta^{\prime}} \ll X e^{-(\log X)^{1 / 4}}
$$

Proof. We can get this lemma from Lemma 11 and Lemma 12 of Tolev [2].

Lemma 8. If $\max \left(|x| / \tau_{1},|y| / \tau_{2}\right)<1$, then

$$
|J(x, y)| \ll X e^{-(\log X)^{1 / 5}},
$$

where

$$
J(x, y)=\sum_{\left|\gamma^{\prime}\right| \leq T} I_{\rho}(x, y), I_{\rho}(x, y)=\int_{\lambda X}^{X} e\left(t^{c} x+t^{d} y\right) t^{\rho-1} \mathrm{~d} t
$$

Proof. Without loss of generality, we consider the case $0 \leq x<\tau_{1}, 0 \leq y<\tau_{2}$. From $\rho=\beta^{\prime}+i \gamma^{\prime}$, we have

$$
I_{\rho}(x, y)=\int_{\lambda X}^{X} t^{\beta^{\prime}-1} e\left(t^{c} x+t^{d} y+\frac{\gamma^{\prime}}{2 \pi} \log t\right) \mathrm{d} t .
$$

Let

$$
F(t)=t^{c} x+t^{d} y+\frac{\gamma^{\prime}}{2 \pi} \log t
$$

We define three sets of nontrivial zeroes of $\zeta(s)$ as

$$
\begin{aligned}
M_{1} & =\left\{\rho:\left|\gamma^{\prime}\right| \leq T,-\gamma^{\prime} / 2 \pi>3\left(c X^{c} x+d X^{d} y\right) / 2\right\}, \\
M_{2} & =\left\{\rho:\left|\gamma^{\prime}\right| \leq T,\left(c(\lambda X)^{c} x+d(\lambda X)^{d} y\right) / 2\right. \\
& \left.\leq-\gamma^{\prime} / 2 \pi \leq 3\left(c X^{c} x+d X^{d} y\right) / 2\right\}, \\
M_{3} & =\left\{\rho:\left|\gamma^{\prime}\right| \leq T,-\gamma^{\prime} / 2 \pi<\left(c(\lambda X)^{c} x+d(\lambda X)^{d} y\right) / 2\right\},
\end{aligned}
$$

and the set $M_{2}$ may be empty.

We first consider $X^{-c} \leq x \leq \tau_{1}$ or $X^{-d} \leq y \leq \tau_{2}$. In this situation, we consider the following three cases.

Case 1. When $\rho \in M_{1}$. In this case, we have

$$
\begin{aligned}
F^{\prime}(t) & =c t^{c-1} x+d t^{d-1} y+\frac{\gamma^{\prime}}{2 \pi t} \\
& \leq \frac{1}{\lambda X}\left(c(\lambda X)^{c} x+d(\lambda X)^{d} y-\frac{-\gamma^{\prime}}{2 \pi}\right)<0 .
\end{aligned}
$$

Applying Lemma 5, we have

$$
\left|I_{\rho}(x, y)\right| \ll \frac{X^{\beta^{\prime}}}{-c X^{c} x-d X^{d} y-\gamma^{\prime} / 2 \pi} \ll \frac{X^{\beta^{\prime}}}{\left|\gamma^{\prime}\right|} .
$$

Therefore, by (46), we can obtain 


$$
\begin{aligned}
& \sum_{\rho \in M_{1}}\left|I_{\rho}(x, y)\right| \ll \sum_{0<\gamma^{\prime} \leq T} \frac{X^{\beta^{\prime}}}{\gamma^{\prime}} \\
& \ll(\log X) \max _{1 \leq T_{1} \leq T}\left(\frac{1}{T_{1}} \sum_{0<\gamma^{\prime} \leq T_{1}} X^{\beta^{\prime}}\right) \\
& \ll X e^{-(\log X)^{1 / 5}} .
\end{aligned}
$$

Case 2. When $\rho \in M_{2}$. In this case, we have

$$
\begin{aligned}
F^{\prime \prime}(t) & =c(c-1) t^{c-2} x+d(d-1) t^{d-2} y-\frac{\gamma^{\prime}}{2 \pi t^{2}} \\
& =\left(X^{c} x+X^{d} y\right) X^{-2}, \\
\left|I_{\rho}(x, y)\right| & \ll \frac{X^{\beta^{\prime}}}{\sqrt{X^{c} x+X^{d} y}} .
\end{aligned}
$$

Hence, we use (45) and get

$$
\begin{aligned}
\sum_{\rho \in M_{2}}\left|I_{\rho}(x, y)\right| & \ll \frac{1}{\sqrt{X^{c} x+X^{d} y}} \sum_{0<\gamma^{\prime} \leq X^{c} x+X^{d} y} X^{\beta^{\prime}} \\
& \ll X e^{-(\log X)^{1 / 4}} .
\end{aligned}
$$

If the set $M_{2}$ is empty, then the upper bound is trivial. Case 3. When $\rho \in M_{3}$. In this case, we have

$$
\begin{aligned}
F^{\prime}(t) & =c t^{c-1} x+d t^{d-1} y+\frac{\gamma^{\prime}}{2 \pi t} \gg \frac{1}{X}\left(c(\lambda X)^{c} x+d(\lambda X)^{d} y-\frac{-\gamma^{\prime}}{2 \pi}\right)>0, \\
\left|I_{\rho}(x, y)\right| & \ll \frac{X^{\beta^{\prime}}}{c(\lambda X)^{c} x+d(\lambda X)^{d} y+\gamma^{\prime} / 2 \pi} .
\end{aligned}
$$

From (46), we have

$$
\begin{aligned}
\sum_{\rho \in M_{3}}\left|I_{\rho}(x, y)\right| \ll & \sum_{-\pi c(\lambda X)^{c} x-\pi} \frac{X^{\beta^{\prime}}}{d(\lambda X)^{d} y<\gamma^{\prime} \leq T} \frac{X^{\beta^{\prime}}}{c(\lambda X)^{c} x+d(\lambda X)^{d} y+\gamma^{\prime} / 2 \pi}+\sum_{X^{c} x+X^{d} y<\gamma^{\prime} \leq T} \frac{X^{\beta^{\prime}}}{\gamma^{\prime}} \\
& \ll \sum_{-\pi c(\lambda X)^{c} x-\pi} \frac{X_{d(\lambda X)^{d} y<\gamma^{\prime} \leq X^{c} x+X^{d} y}^{X^{c} x+X^{d} y}}{\ll}(\log X) \max _{1 \leq T_{1} \leq T}\left(\frac{1}{T_{1}} \sum_{0<\gamma^{\prime} \leq T_{1}} X^{\beta^{\prime}}\right) \\
& \ll X e^{-(\log X)^{1 / 5}} .
\end{aligned}
$$

Therefore, from (54)-(58), we obtain

$$
|J(x, y)| \ll X e^{-(\log X)^{1 / 5}} .
$$

Now we consider the remaining case $0 \leq x \leq X^{-c}$ and $0 \leq y \leq X^{-d}$. We use the trivial estimate of $\left|I_{\rho}(x, y)\right|$ and get

$$
\sum_{\rho \in M_{2}}\left|I_{\rho}(x, y)\right| \ll \sum_{\rho \in M_{2}} X^{\beta^{\prime}} \ll X^{\beta_{0}}
$$

where $\quad \beta_{0}=\max _{\rho \in M_{2}} \beta^{\prime}<1 . \quad \sum_{\rho \in M_{1}}\left|I_{\rho}(x, y)\right| \quad$ and $\sum_{\rho \in M_{3}}\left|I_{\rho}(x, y)\right|$ are estimated analogically as in the previous case. Then, the estimate for $|J(x, y)|$ is established. 
Lemma 9. If $\max \left(|x| / \tau_{1},|y| / \tau_{2}\right)<1$, then

$$
S(x, y)=I(x, y)+O\left(X e^{-(\log X)^{1 / 5}}\right)
$$

$$
I(x, y)=\int_{\lambda X}^{X} e\left(x t^{c}+y t^{d}\right) \mathrm{d} t .
$$

where

Proof. Noting

$$
\sum_{\substack{\lambda X<p^{v} \leq X \\ v>1}}(\log p) e\left(p^{c v} x+p^{d v} y\right) \ll \sum_{p^{2} \leq X} \log p \sum_{\frac{\log (\lambda X)}{\log p}<<<\frac{\log X}{\log p}} 1 \ll X^{1 / 2}
$$

we have

$$
\begin{aligned}
S(x, y) & =\sum_{\lambda X<n \leq X} \Lambda(n) e\left(n^{c} x+n^{d} y\right)-\sum_{\substack{\lambda X<p^{v} \leq X \\
v>1}}(\log p) e\left(p^{c v} x+p^{d v} y\right) \\
& =\sum_{\lambda X<n \leq X} \Lambda(n) e\left(n^{c} x+n^{d} y\right)+O\left(X^{1 / 2}\right):=U(x, y)+O\left(X^{1 / 2}\right),
\end{aligned}
$$

where $v$ denotes an integer number. Applying Abel's Then, Lemma 6 implies transformation, we get

$$
\begin{aligned}
U(x, y)= & -\int_{\lambda X}^{X}(\Psi(t)-\Psi(\lambda X)) \frac{\mathrm{d}}{\mathrm{d} t}\left(e\left(t^{c} x+t^{d} y\right)\right) \mathrm{d} t \\
& +(\Psi(X)-\Psi(\lambda X)) e\left(X^{c} x+X^{d} y\right) .
\end{aligned}
$$

$$
\begin{aligned}
U(x, y)= & -\int_{\lambda X}^{X}\left(t-\lambda X-\sum_{\left|\gamma^{\prime}\right| \leq T} \frac{t^{\rho}-(\lambda X)^{\rho}}{\rho}+O\left(\frac{X \log ^{2} X}{T}\right)\right) \frac{\mathrm{d}}{\mathrm{d} t}\left(e\left(t^{c} x+t^{d} y\right)\right) \mathrm{d} t \\
& +\left(X-\lambda X-\sum_{\left|\gamma^{\prime}\right| \leq T} \frac{X^{\rho}-(\lambda X)^{\rho}}{\rho}+O\left(\frac{X \log ^{2} X}{T}\right)\right) e\left(X^{c} x+X^{d} y\right) \\
= & -\int_{\lambda X}^{X}\left(t-\lambda X-\sum_{\left|\gamma^{\prime}\right| \leq T} \frac{t^{\rho}-(\lambda X)^{\rho}}{\rho}\right) \frac{\mathrm{d}}{\mathrm{d} t}\left(e\left(t^{c} x+t^{d} y\right)\right) \mathrm{d} t \\
& +\left(X-\lambda X-\sum_{\left|\gamma^{\prime}\right| \leq T} \frac{X^{\rho}-(\lambda X)^{\rho}}{\rho}\right) e\left(X^{c} x+X^{d} y\right)+O\left(\frac{X \log ^{2} X\left(X^{c} \tau_{1}+X^{d} \tau_{2}\right)}{T}\right) .
\end{aligned}
$$

Using integration by parts, we have 


$$
\begin{aligned}
& U(x, y)=\int_{\lambda X}^{X} e\left(t^{c} x+t^{d} y\right)\left(1-\sum_{\left|\gamma^{\prime}\right| \leq T} t^{\rho-1}\right) \mathrm{d} t+O\left(\frac{X \log ^{2} X\left(X^{c} \tau_{1}+X^{d} \tau_{2}\right)}{T}\right) \\
& =I(x, y)-J(x, y)+O\left(\frac{X \log ^{2} X\left(X^{c} \tau_{1}+X^{d} \tau_{2}\right)}{T}\right) . \\
& H=\int_{-\infty}^{\infty} \int_{-\infty}^{\infty} I^{6}(x, y) e\left(-N_{1} x-N_{2} y\right) \varphi_{\varepsilon_{1}}(x) \varphi_{\varepsilon_{2}}(y) \mathrm{d} x \mathrm{~d} y,
\end{aligned}
$$

From Lemma 8, this lemma follows.

Lemma 10 (see [18], Lemma 8). For $S(x, y)$ defined by (17), we have

and

$$
\iint_{\Omega_{1}}|S(x, y)|^{4} \varphi_{\varepsilon_{1}}(x) \varphi_{\varepsilon_{2}}(y) \mathrm{d} x \mathrm{~d} y \ll \varepsilon_{1} \varepsilon_{2} X^{4-c-d} \log ^{8} X .
$$

$$
H_{1}=\iint_{\Omega_{1}} I^{6}(x, y) e\left(-N_{1} x-N_{2} y\right) \varphi_{\varepsilon_{1}}(x) \varphi_{\varepsilon_{2}}(y) \mathrm{d} x \mathrm{~d} y .
$$

Lemma 11 (see [18], Lemma 9). For $I(x, y)$ defined in Lemma 9, we have

$$
\int_{-\infty}^{\infty} \int_{-\infty}^{\infty}|I(x, y)|^{4} \varphi_{\varepsilon_{1}}(x) \varphi_{\varepsilon_{2}}(y) \mathrm{d} x \mathrm{~d} y \ll \varepsilon_{1} \varepsilon_{2} X^{4-c-d} \log ^{4} X .
$$

Lemma 12. For integrals $H$ and $H_{1}$ defined by (70) and (71), respectively, we have

$$
\left|H-H_{1}\right| \ll \frac{\varepsilon_{1} \varepsilon_{2} X^{6-c-d}}{\log X} .
$$

Write

Proof. We have

$$
\begin{aligned}
\left|H-H_{1}\right| & \ll \iint_{\mathbb{R}^{2} / \Omega_{1}}|I(x, y)|^{6} \varphi_{\varepsilon_{1}}(x) \varphi_{\varepsilon_{2}}(y) \mathrm{d} x \mathrm{~d} y \\
& \ll \max _{\mathbb{R}^{2} \backslash \Omega_{1}}|I(x, y)|^{2} \iint_{\mathbb{R}^{2}}|I(x, y)|^{4} \varphi_{\varepsilon_{1}}(x) \varphi_{\varepsilon_{2}}(y) \mathrm{d} x \mathrm{~d} y .
\end{aligned}
$$

Applying Lemma 5 with $G(t)=1$ and $F(t)=x t^{c}+y t^{d}$, from $F^{\prime \prime}(t)=c(c-1) x t^{c-2}+d(d-1) y t^{d-2}$, we can get

$$
\max _{\mathbb{R}^{2} \backslash \Omega_{1}}|I(x, y)| \ll X^{5 / 8+\eta / 2} .
$$

Then, by Lemma 11, we can get this lemma.
Lemma 13. For the integral $H$ defined by (70), we have

$$
H \gg \varepsilon_{1} \varepsilon_{2} X^{6-c-d} \text {. }
$$

Proof. We have

$$
\begin{aligned}
H & =\int_{\lambda X<t_{1}, \ldots, t_{6} \leq X} \cdots \int_{-\infty}^{\infty} \int_{-\infty}^{\infty} e\left(x\left(t_{1}^{c}+\cdots+t_{6}^{c}-N_{1}\right)\right) e\left(y\left(t_{1}^{d}+\cdots+t_{6}^{d}-N_{2}\right)\right) \times \varphi_{\varepsilon_{1}}(x) \varphi_{\varepsilon_{2}}(y) \mathrm{d} x \mathrm{~d} y \mathrm{~d} t_{1} \cdots \mathrm{d} t_{6} \\
& =\int \cdots \int \Delta \mathrm{d} t_{1} \cdots \mathrm{d} t_{6}+\int_{C_{1}} \cdots \int \Delta \mathrm{d} t_{1} \cdots \mathrm{d} t_{6}
\end{aligned}
$$

where 


$$
\begin{aligned}
C_{1} & =\left\{\lambda X<t_{1}, \ldots, t_{6} \leq X:\left|\frac{t_{1}^{c}+\cdots+t_{6}^{c}-N_{1}}{\varepsilon_{1}}\right|<1 \text { and }\left|\frac{t_{1}^{d}+\cdots+t_{6}^{d}-N_{2}}{\varepsilon_{2}}\right|<1\right\}, \\
C_{2} & =\left\{\lambda X<t_{1}, \ldots, t_{6} \leq X:\left|\frac{t_{1}^{c}+\cdots+t_{6}^{c}-N_{1}}{\varepsilon_{1}}\right| \geq 1 \text { or }\left|\frac{t_{1}^{d}+\cdots+t_{6}^{d}-N_{2}}{\varepsilon_{2}}\right| \geq 1\right\}, \\
\Delta & =\int_{-\infty}^{\infty} \int_{-\infty}^{\infty} e\left(x\left(t_{1}^{c}+\cdots+t_{6}^{c}-N_{1}\right)\right) e\left(y\left(t_{1}^{d}+\cdots+t_{6}^{d}-N_{2}\right)\right) \varphi_{\varepsilon_{1}}(x) \varphi_{\varepsilon_{2}}(y) \mathrm{d} x \mathrm{~d} y .
\end{aligned}
$$

From (31), we get

$$
\begin{aligned}
& \int_{-\infty}^{\infty} e\left(x\left(t_{1}^{c}+\cdots+t_{6}^{c}-N_{1}\right)\right) \varphi_{\varepsilon_{1}}(x) \mathrm{d} x=\varphi\left(\frac{t_{1}^{c}+\cdots+t_{6}^{c}-N_{1}}{\varepsilon_{1}}\right), \\
& \int_{-\infty}^{\infty} e\left(x\left(t_{1}^{d}+\cdots+t_{6}^{d}-N_{2}\right)\right) \varphi_{\varepsilon_{2}}(x) \mathrm{d} x=\varphi\left(\frac{t_{1}^{d}+\cdots+t_{6}^{d}-N_{2}}{\varepsilon_{2}}\right) .
\end{aligned}
$$

In (76), the integral over $C_{2}$ is convergent, and hence $\int \cdots \int \Delta \mathrm{d} t_{1} \cdots \mathrm{d} t_{6}=O(1)$. Applying Lemma 2, we have $H \gg \int_{C_{1}}^{C_{2}} \ldots \int 1 \mathrm{~d} t_{1} \cdots \mathrm{d} t_{6} \gg X^{6} \int \cdots \int_{C_{1}^{\prime}} 1 \mathrm{~d}\left(t_{1} / X\right) \cdots \mathrm{d}\left(t_{6} / X\right)$,

where

$$
\begin{aligned}
C_{1}^{\prime}= & \left\{\lambda<t_{1} / X, \ldots, t_{6} / X:\left|\left(t_{1} / X\right)^{c}+\cdots+\left(t_{6} / X\right)^{c}-1\right|\right. \\
& <\varepsilon_{1} X^{-c} \text { and }\left|\left(t_{1} / X\right)^{d}+\cdots+\left(t_{6} / X\right)^{d}-N_{2} / X^{d}\right| \\
& \left.<\varepsilon_{2} X^{-d}\right\} .
\end{aligned}
$$

From (13) and Lemma 1, we have

$$
H \gg \varepsilon_{1} \varepsilon_{2} X^{6-c-d} .
$$

Lemma 14. For the integral $D_{1}$ in (20), we have

$$
\left|D_{1}\right| \gg \varepsilon_{1} \varepsilon_{2} X^{6-c-d} \text {. }
$$

Proof. We have

$$
\begin{aligned}
\left|D_{1}-H_{1}\right| & \ll \iint_{\Omega_{1}}\left|S^{6}(x, y)-I^{6}(x, y)\right| \varphi_{\varepsilon_{1}}(x) \varphi_{\varepsilon_{2}}(y) \mathrm{d} x \mathrm{~d} y \\
& \ll \max _{\Omega_{1}}|S(x, y)-I(x, y)|^{2} \iint_{\Omega_{1}}\left(|S(x, y)|^{4}+|I(x, y)|^{4}\right) \varphi_{\varepsilon_{1}}(x) \varphi_{\varepsilon_{2}}(y) \mathrm{d} x \mathrm{~d} y .
\end{aligned}
$$

From Lemmas 9-11, we can obtain

$$
\left|D_{1}-H_{1}\right| \ll \frac{\varepsilon_{1} \varepsilon_{2} X^{6-c-d}}{\log X} .
$$

By Lemma 12, we have

$$
D_{1}=H+O\left(\frac{\varepsilon_{1} \varepsilon_{2} X^{6-c-d}}{\log X}\right)
$$

Then, from Lemma 13, we can get this lemma.

\section{The Estimate for the Integral $D_{2}$}

Lemma 15. Let $a_{m}, b_{n}$ be arbitrary complex numbers and $\max \left(|x| / \tau_{1},|y| / \tau_{2}\right) \geq 1, \max \left(|x| / K_{1},|y| / K_{2}\right) \leq 1$,

$$
X^{1 / 4}<R \leq X^{1 / 2}, X^{1 / 4}<L<L_{1} \leq 2 L, \text { LR } \leq X .
$$

Write

$$
W=\sum_{L<n \leq L_{1}} \sum_{X^{1 / 4}<m \leq R} a_{m} b_{n} e\left((m n)^{c} x+(m n)^{d} y\right) .
$$


Then,

$$
|W| \ll(\mathscr{B})^{1 / 2} X^{101 / 238} \log ^{-13} X,
$$

where

$$
\mathscr{A}=\sum_{X^{1 / 4}<m \leq R}\left|a_{m}\right|^{2}, \mathscr{B}=\sum_{L<n \leq L_{1}}\left|b_{n}\right|^{2}
$$

Proof. Without loss of generality, we can consider the case $\tau_{1} \leq x \leq K_{1}, 0<y \leq K_{2}$. We define $R_{i}(0 \leq i \leq Q)$ :

$$
R_{0}=X^{1 / 4}, R_{i+1}=\min \left(R_{i}+s, R\right), R_{Q}=R,
$$

$$
W=\sum_{L<n \leq L_{1}} \sum_{1 \leq i \leq Q} \sum_{R_{i-1}<m \leq R_{i}} a_{m} b_{n} e\left((m n)^{c} x+(m n)^{d} y\right) .
$$

By Cauchy's inequality, we have

$$
\begin{aligned}
|W|^{2} \leq & \mathscr{B} Q \sum_{L<n \leq L_{1}} \sum_{1 \leq i \leq Q} \sum_{R_{i-1}<m_{1}, m_{2} \leq R_{i}} a_{m_{1}} \bar{a}_{m_{2}} e\left(\left(m_{1}^{c}-m_{2}^{c}\right) n^{c} x\right. \\
& \left.+\left(m_{1}^{d}-m_{2}^{d}\right) n^{d} y\right) .
\end{aligned}
$$

We rearrange the sums as follows:

where $s \in[1, R]$ will be determined later and $Q \ll R / s$. Thus, $W$ can be rewritten as

$$
\begin{aligned}
|W|^{2} \ll & \mathscr{B} Q\left(\sum_{L<n \leq L_{1}} \sum_{1 \leq i \leq Q} \sum_{R_{i-1}<m \leq R_{i}}\left|a_{m}\right|^{2}\right) \\
& +\mathscr{B} Q \sum_{\substack { 1 \leq i \leq Q \\
\begin{subarray}{c}{R_{i-1}<m_{1}, m_{2} \leq R_{i} \\
m_{1} \neq m_{2}{ 1 \leq i \leq Q \\
\begin{subarray} { c } { R _ { i - 1 } < m _ { 1 } , m _ { 2 } \leq R _ { i } \\
m _ { 1 } \neq m _ { 2 } } }\end{subarray}}\left(\left|a_{m_{1}}\right|\left|a_{m_{2}}\right|\left|\sum_{L<n \leq L_{1}} e\left(\left(m_{1}^{c}-m_{2}^{c}\right) n^{c} x+\left(m_{1}^{d}-m_{2}^{d}\right) n^{d} y\right)\right|\right) \\
& \ll \mathscr{B} Q\left(\mathscr{A} L+\sum_{1 \leq h \leq s} \sum_{1 \leq i \leq Q} \sum_{R_{i-1}<m \leq R_{i}-h}\left|a_{m}\right|\left|a_{m+h}\right|\left|\sum_{L<n \leq L_{1}} e(f(n))\right|\right),
\end{aligned}
$$

where

$$
f(n)=\left((m+h)^{c}-m^{c}\right) n^{c} x+\left((m+h)^{d}-m^{d}\right) n^{d} y .
$$

Next, we handle the exponential sum $\left|\sum_{L<n \leq L_{1}} e(f(n))\right|$ of (93). From the derivatives of $f$, we get

$$
\begin{aligned}
f^{j}(n) \asymp & {\left[\left((m+h)^{c}-m^{c}\right) L^{c-1} x\right.} \\
& \left.+\left((m+h)^{d}-m^{d}\right) L^{d-1} y\right] L^{1-j}
\end{aligned}
$$

Then, by Lemma 3 with the exponent pair (see also [21], p. 77)

$$
B A^{2} B A^{2} B(0,1)=(13 / 40,22 / 40),
$$
we have

$$
\begin{aligned}
\left|\sum_{L<n \leq L_{1}} e(f(n))\right| & \ll\left[\left((m+h)^{c}-m^{c}\right) L^{c-1} x+\left((m+h)^{d}-m^{d}\right) L^{d-1} y\right]^{13 / 40} L^{22 / 40} \\
& \ll\left[\left((m+h)^{c}-m^{c}\right) L^{c-1} K_{1}+\left((m+h)^{d}-m^{d}\right) L^{d-1} K_{2}\right]^{13 / 40} L^{22 / 40} \\
& \ll s^{13 / 40}\left(R^{13(c-1) / 40} L^{(13 c+9) / 40} K_{1}^{13 / 40}+R^{13(d-1) / 40} L^{(13 d+9) / 40} K_{2}^{13 / 40}\right) .
\end{aligned}
$$

Inserting this upper bound into (93), we have 


$$
\begin{aligned}
|W|^{2} \ll & \mathscr{B} Q\left[\mathscr{A} L+s^{13 / 40}\left(R^{13(c-1) / 40} L^{(13 c+9) / 40} K_{1}^{13 / 40}\right.\right. \\
& \left.\left.+R^{13(d-1) / 40} L^{(13 d+9) / 40} K_{2}^{13 / 40}\right) \sum_{0}\right]
\end{aligned}
$$

where

$$
\sum_{0} \sum_{1 \leq h \leq s} \sum_{1 \leq i \leq Q} \sum_{R_{i-1}<m \leq R_{i}-h}\left|a_{m}\right|\left|a_{m+h}\right|
$$

By Cauchy's inequality, we obtain

$$
\begin{aligned}
\sum_{0} & \leq \sum_{1 \leq h \leq s} \sum_{X^{1 / 4}<m \leq R-h}\left|a_{m}\right|\left|a_{m+h}\right| \\
& \leq \sum_{1 \leq h \leq s}\left(\sum_{X^{1 / 4}<m \leq R-h}\left|a_{m}\right|^{2}\right)^{1 / 2}\left(\sum_{X^{1 / 4}<m \leq R-h}\left|a_{m+h}\right|^{2}\right)^{1 / 2} \\
& \ll s \mathscr{A} .
\end{aligned}
$$

From (98) and $Q \ll R / s$, we have

$$
\begin{aligned}
|W|^{2} \ll & \mathscr{A} \mathscr{B} L R\left[1 / s+s^{13 / 40}\left(R^{13(c-1) / 40} L^{(13 c-31) / 40} K_{1}^{13 / 40}\right.\right. \\
& \left.\left.+R^{13(d-1) / 40} L^{(13 d-31) / 40} K_{2}^{13 / 40}\right)\right] .
\end{aligned}
$$

We take $1 / s=s^{13 / 40}\left(R^{13(c-1) / 40} L^{(13 c-31) / 40} K_{1}^{13 / 40}+\right.$ $\left.R^{13(d-1) / 40} L^{(13 d-31) / 40} K_{2}^{13 / 40}\right)$, i.e.,

$$
\begin{aligned}
s= & R^{13(1-c) / 53} L^{(31-13 c) / 53} K_{1}^{-13 / 53} \\
& +R^{13(1-d) / 53} L^{(31-13 d) / 53} K_{2}^{-13 / 53},
\end{aligned}
$$

and then $s \in[1, R]$. Inserting this value of $s$ into (101), we get

$$
|W|^{2} \ll \mathscr{A} \mathscr{B} X^{101 / 119} \log ^{-26} X,
$$

which yields this lemma.

Lemma 16 (see [2], Lemma 9). Let $a_{m}, b_{n}$ be arbitrary complex numbers and $L<L_{1} \leq 2 L, L \leq X$. Write

$$
V=\sum_{L<n \leq L_{1}} \sum_{X^{1 / 4}<m \leq X / n} a_{m} b_{n} e\left((m n)^{c} x+(m n)^{d} y\right) .
$$

Then, there exist $a_{m}^{\prime}, b_{n}^{\prime}$ satisfying $\left|a_{m}^{\prime}\right| \leq\left|a_{m}\right|,\left|b_{n}^{\prime}\right| \leq\left|b_{n}\right|$ such that

$$
|V| \ll(\log X)\left|\sum_{L<n \leq L_{1}} \sum_{X^{1 / 4}<m \leq X / L} a_{m}^{\prime} b_{n}^{\prime} e\left((m n)^{c} x+(m n)^{d} y\right)\right| .
$$

Lemma 17. Assume that $\max \left(|x| / \tau_{1},|y| / \tau_{2}\right)$ $\geq 1, \max \left(|x| / K_{1},|y| / K_{2}\right) \leq 1$; then,

$$
|S(x, y)| \ll X^{110 / 119} \log ^{-9} X \text {. }
$$

Proof. Without loss of generality, we can consider the case $\tau_{1} \leq x \leq K_{1}, 0<y \leq K_{2}$. Clearly,

$$
S(x, y)=V_{0}(x, y)-V_{1}(x, y)+O\left(X^{1 / 2}\right)
$$

where

$$
\begin{aligned}
& V_{0}(x, y)=\sum_{X^{1 / 4}<n \leq X} \Lambda(n) e\left(n^{c} x+n^{d} y\right), \\
& V_{1}(x, y)=\sum_{X^{1 / 4}<n \leq \lambda X} \Lambda(n) e\left(n^{c} x+n^{d} y\right) .
\end{aligned}
$$

Hence, it is sufficient to prove that

$$
\left|V_{0}(x, y)\right|,\left|V_{1}(x, y)\right| \ll X^{110 / 119} \log ^{-9} X .
$$

The estimates of $V_{0}(x, y)$ and $V_{1}(x, y)$ are similar, and thus we focus on the estimate of $V_{0}(x, y)$. Using Vaughan's identity (see [23]), we have

$$
V_{0}(x, y)=S_{1}-S_{2}-S_{3},
$$

where

$$
\begin{aligned}
& S_{1}=\sum_{g \leq X^{1 / 4}} \mu(g) \sum_{l \leq X / g}(\log l) e\left((l g)^{c} x+(l g)^{d} y\right), \\
& S_{2}=\sum_{k \leq X^{1 / 2}} \sum_{r \leq X / k} c_{k} e\left((k r)^{c} x+(k r)^{d} y\right), \\
& S_{3}=\sum_{X^{1 / 4}<m \leq X^{3 / 4}} \sum_{X^{1 / 4}<n \leq X / m} a_{m} \Lambda(n) e\left((m n)^{c} x+(m n)^{d} y\right),
\end{aligned}
$$

where $\left|c_{k}\right| \leq \log k$ and $\left|a_{m}\right| \leq \tau(m)$.

For $S_{2}$, noting

$$
\sum_{X^{1 / 4}<k \leq X^{1 / 2}} \sum_{r \leq X^{1 / 4}} c_{k} e\left((k r)^{c} x+(k r)^{d} y\right) \ll X^{3 / 4} \log X,
$$

we have

$$
S_{2}=S_{2}^{(1)}+S_{2}^{(2)}+O\left(X^{3 / 4} \log X\right)
$$

where

$$
\begin{aligned}
& S_{2}^{(1)}=\sum_{k \leq X^{1 / 4}} \sum_{r \leq X / k} c_{k} e\left((k r)^{c} x+(k r)^{d} y\right), \\
& S_{2}^{(2)}=\sum_{X^{1 / 4}<k \leq X^{1 / 2} X^{1 / 4}<r \leq X / k} c_{k} e\left((k r)^{c} x+(k r)^{d} y\right) .
\end{aligned}
$$

For $S_{1}$, we have

$$
\left|S_{1}\right| \leq \sum_{g \leq X^{1 / 4}}\left|\sum_{l \leq X / g}(\log l) e\left((\lg )^{c} x+(\lg )^{d} y\right)\right| .
$$

The second summation over $l$ in (115) is

$$
\ll\left(\log ^{2} X\right) \max _{L_{2} \in\left[L, L_{1}\right]}\left|\sum_{L<l \leq L_{2}} e(h(l))\right|,
$$

where $L<L_{1} \leq 2 L, L_{1} \leq X / g$, and $h(l)=(l g)^{c} x+(\lg )^{d} y$. Noting $h^{j}(l) \asymp\left(L^{c-1} g^{c} x+L^{d-1} g^{d} y\right) L^{1-j}$, Lemma 3 with exponent pair $(13 / 40,22 / 40)$ gives 


$$
\begin{aligned}
\left|\sum_{L<l \leq L_{1}} e(h(l))\right| & \ll\left(L^{c-1} g^{c} K_{1}+L^{d-1} g^{d} K_{2}\right)^{13 / 40} L^{22 / 40} \\
& \ll X^{13 c / 40} L^{9 / 40} K_{1}^{13 / 40}+X^{13 d / 40} L^{9 / 40} K_{2}^{13 / 40} .
\end{aligned}
$$

Recalling (115) and (116), we have

$$
\left|S_{1}\right| \ll X^{110 / 119} \log ^{-9} X
$$

Arguing similarly, we can get the following same bound of $S_{2}^{(1)}$ :

$$
\left|S_{2}^{(1)}\right| \ll X^{110 / 119} \log ^{-9} X .
$$

For $S_{3}$, we divide it into three parts:

$$
S_{3}=W_{1}+W_{2}+W_{3}
$$

where

$$
\begin{aligned}
& W_{1}=\sum_{X^{1 / 2}<n \leq X^{3 / 4} X^{1 / 4}<m \leq X / n} \sum_{m} a_{m} \Lambda(n) e\left((m n)^{c} x+(m n)^{d} y\right), \\
& W_{2}=\sum_{X^{1 / 2}<n \leq X^{3 / 4} X^{1 / 4}<m \leq X / n} a_{n} \Lambda(m) e\left((m n)^{c} x+(m n)^{d} y\right), \\
& W_{3}=\sum_{X^{1 / 4}<n \leq X^{1 / 2}} \sum_{X^{1 / 4}<m \leq X^{1 / 2}} a_{m} \Lambda(n) e\left((m n)^{c} x+(m n)^{d} y\right) .
\end{aligned}
$$
have

For $W_{1}$, using dyadic subdivision and Lemma 16 , we

$$
\left|W_{1}\right| \ll\left(\log ^{2} X\right)\left|W_{1}^{\prime}(L)\right|
$$

where

$$
W_{1}^{\prime}(L)=\sum_{L<n \leq L_{1}} \sum_{X^{1 / 4}<m \leq X / L} a_{m}^{\prime} b_{n}^{\prime} e\left((m n)^{c} x+(m n)^{d} y\right),
$$

where $\quad X^{1 / 2} \leq L<L_{1} \leq 2 L \leq X^{3 / 4},\left|a_{m}^{\prime}\right| \leq\left|a_{m}\right| \leq \tau(m)$, and $\left|b_{n}^{\prime}\right| \leq \Lambda(n)$. Lemma 15 implies

$$
\begin{aligned}
\left|W_{1}^{\prime}(L)\right| & \ll\left(\frac{X}{L} \log ^{3} X \cdot L \log X\right)^{1 / 2} X^{101 / 238} \log ^{-13} X \\
& =X^{110 / 119} \log ^{-11} X,
\end{aligned}
$$

where we used the mean value estimates

$$
\sum_{m \leq y} \tau^{2}(m) \ll y \log ^{3} y, \sum_{n \leq y} \Lambda^{2}(n) \ll y \log y .
$$

Therefore, we have

$$
\left|W_{1}\right| \ll X^{110 / 119} \log ^{-9} X .
$$

We estimate $W_{2}$ and $W_{3}$ similar to $W_{1}$ and get

$$
\left|W_{2}\right|,\left|W_{3}\right| \ll X^{110 / 119} \log ^{-9} X \text {. }
$$

For $S_{2}^{(2)}$, we follow a similar argument to $S_{3}$ and get

$$
S_{2}^{(2)}=U_{1}+U_{2}
$$

where

$$
\begin{aligned}
& U_{1}=\sum_{X^{1 / 4}<r \leq X^{1 / 2}} \sum_{X^{1 / 4}<k \leq X^{1 / 2}} c_{k} e\left((k r)^{c} x+(k r)^{d} y\right), \\
& U_{2}=\sum_{X^{1 / 2}<r \leq X^{3 / 4}} \sum_{X^{1 / 4}<k \leq X / r} c_{k} e\left((k r)^{c} x+(k r)^{d} y\right) .
\end{aligned}
$$

Using Lemmas 15 and 16 and the mean value estimate

$$
\sum_{k \leq y} \log ^{2} k \ll y \log ^{2} y
$$

we get

$$
\left|U_{1}\right|,\left|U_{2}\right| \ll X^{110 / 119} \log ^{-9} X
$$

From (110) and (118)-(131), we can obtain (109).

Lemma 18 (see [18], Lemma 14). For $S(x, y)$ defined by (17), we have

$$
\int_{-\infty}^{\infty} \int_{-\infty}^{\infty}|S(x, y)|^{4} \varphi_{\varepsilon_{1}}(x) \varphi_{\varepsilon_{2}}(y) \mathrm{d} x \mathrm{~d} y \ll X^{2} \log ^{6} X .
$$

Lemma 19. For the integral $D_{2}$ in (20), we have

$$
\left|D_{2}\right| \ll \frac{\varepsilon_{1} \varepsilon_{2} X^{6-c-d}}{\log X} .
$$

Proof. By Lemmas 17 and 18, we have

$$
\begin{aligned}
\left|D_{2}\right| & \ll \max _{\Omega_{2}}|S(x, y)|^{2} \int_{-\infty}^{\infty} \int_{-\infty}^{\infty}|S(x, y)|^{4} \varphi_{\varepsilon_{1}}(x) \varphi_{\varepsilon_{2}}(y) \mathrm{d} x \mathrm{~d} y \\
& \ll\left(X^{110 / 119} \log ^{-9} X\right)^{2} X^{2} \log ^{6} X \\
& \ll X^{458 / 119} \log ^{-12} X,
\end{aligned}
$$

which yields this lemma.

\section{Proof of Theorem 1}

In this section, we first give the estimate of $D_{3}$ in (20) and then complete the proof of Theorem 1 .

For the integral $D_{3}$ in (20), by Lemma 2, we can easily get

$$
\left|D_{3}\right| \ll 1 \text {. }
$$

From Lemmas 14 and 19 and (135), we know that (22)-(24) follows, respectively. Therefore, recalling Lemma 4 and (20), we can get

$$
B \gg \varepsilon_{1} \varepsilon_{2} X^{6-c-d},
$$

from which we complete the proof of Theorem 1 . 


\section{Data Availability}

The data supporting the findings of this study are included within the article.

\section{Conflicts of Interest}

The authors declare that they have no conflicts of interest.

\section{Acknowledgments}

This study was supported by the National Natural Science Foundation of China (grant nos. 11771256 and 11801328).

\section{References}

[1] I. I. Piatetski-Shapiro, "On a variant of Waring-Goldbach's problem," Matematicheskii Sbornik, vol. 30, no. 1, pp. 105120, 1952, in Russian.

[2] D. Tolev, "On a diophantine inequality involving prime numbers," Acta Arithmetica, vol. 61, no. 3, pp. 289-306, 1992.

[3] R. Baker and A. Weingartner, "A ternary Diophantine inequality over primes," Acta Arithmetica, vol. 162, no. 2, pp. 159-196, 2014.

[4] Y. C. Cai, "On a Diophantine inequality involving prime numbers," Acta Mathematica Sinica (Chinese Series), vol. 39, no. 6, pp. 733-742, 1996.

[5] Y. Cai, "On a diophantine inequality involving prime numbers (III)," Acta Mathematica Sinica, vol. 15, no. 3, pp. 387-394, 1999.

[6] Y. Cai, "A ternary Diophantine inequality involving primes," International Journal of Number Theory, vol. 14, no. 08, pp. 2257-2268, 2018.

[7] X. D. Cao and W. G. Zhai, "A Diophantine inequality with prime numbers," Acta Mathematica Sinica (Chinese Series), vol. 45, no. 2, pp. 361-370, 2002.

[8] A. Kumchev, "A Diophantine inequality involving prime powers," Acta Arithmetica, vol. 89, no. 4, pp. 311-330, 1999.

[9] A. Kumchev and T. Nedeva, "On an equation with prime numbers," Acta Arithmetica, vol. 83, no. 2, pp. 117-126, 1998.

[10] R. C. Baker and A. Weingartner, "Some applications of the double large sieve," Monatshefte für Mathematik, vol. 170, no. 3-4, pp. 261-304, 2013.

[11] J. Huang and H. F. Liu, "Diophantine approximation with mixed powers of primes," Taiwanese Journal of Mathematics, vol. 23, no. 5, pp. 1073-1090, 2019.

[12] A. V. Kumchev, "A binary additive equation involving fractional powers," International Journal of Number Theory, vol. 05, no. 02, pp. 281-292, 2009.

[13] S. Li and Y. Cai, "On a Diophantine inequality involving prime numbers," The Ramanujan Journal, vol. 52, no. 1, pp. 163-174, 2020.

[14] T. Li and H. Liu, "Diophantine approximation over PiatetskiShapiro primes," Journal of Number Theory, vol. 211, pp. 184-198, 2020.

[15] H. Liu, "Diophantine approximation with one prime, two squares of primes and powers of two," The Ramanujan Journal, vol. 51, no. 1, pp. 85-97, 2020.

[16] W. G. Zhai and X. D. Cao, "On a binary Diophantine inequality," Advances in Mathematics (China), vol. 32, no. 6, pp. 706-721, 2003.

[17] W. Zhai and X. Cao, "On a diophantine inequality over primes (II)," Monatshefte für Mathematik, vol. 150, no. 2, pp. 173-179, 2007.
[18] D. Tolev, "On a system of two diophantine inequalities with prime numbers," Acta Arithmetica, vol. 69, no. 4, pp. 387-400, 1995.

[19] W. Zhai, "On a system of two diophantine inequalities with prime numbers," Acta Arithmetica, vol. 92, no. 1, pp. 31-46, 2000.

[20] W. G. Zhai and X. D. Cao, "On systems of Diophantine inequalities and equations with prime numbers," Advances in Mathematics (China), vol. 31, no. 3, pp. 243-248, 2002.

[21] A. Ivić, The Riemann Zeta-Function, Theory and Applications, Dover Publicatios, Inc., Mineola, NY, USA, 2003.

[22] A. A. Karatsuba, Principles of Analytic Number Theory, Nauka, Moscow, Russia, in Russian, 2nd edition, 1983.

[23] R. C. Vaughan, "On the distribution of $\alpha \mathrm{p}$ modulo 1," Mathematika, vol. 24, no. 2, pp. 135-141, 1977. 